\title{
Incompressible states in double quantum dots
}

\author{
N. Barberán and J. Soto \\ Departament d'Estructura i Constituents de la Matèria, Facultat de Física, Universitat de Barcelona, E-08028 Barcelona, \\ Catalonia, Spain
}

(Received 4 October 2002; revised manuscript received 17 January 2003; published 11 July 2003)

\begin{abstract}
Incompressible (magic) states that result from many-body effects in vertically coupled quantum dots submitted to strong magnetic fields such that only the lowest Landau level is relevant are studied within an exact diagonalization calculation for $N=3,5$, and 6 , electrons. We find that the sequences of total angular momentum $M$ for which these incompressible states exist depend on the interplay between the interdot hopping parameter $\Delta_{t}$ and the interdot distance $d$. For $d$ of the order of the magnetic length and for all values of $\Delta_{t}$, we conclude that, in contrast to previous claims, these incompressible states appear at magic values of $M$ which do not differ from those obtained for a single dot: namely, $M=N(N-1) / 2+j N$, where $j$ is a positive integer number. For large interdot distance and simultaneously small interdot hopping parameter, new sequences of magic values of $M$ are observed. These new sequences can be easily understood in terms of a transition regime towards a system of two decoupled single dots. However, important differences in the nature of the incompressible ground states are found with respect to those of a single dot.
\end{abstract}

DOI: 10.1103/PhysRevB.68.045306

PACS number(s): 73.21.La, 73.43.-f, 73.43.Lp

\section{INTRODUCTION}

Much effort has been devoted to understand the magic incompressible states (IS's) of two-dimensional electronic nanostructures. This is due to the fact that they are closely related to the states that determine properties like superconductivity or the quantum Hall effect $(\mathrm{QHE}),{ }^{1,2}$ which are striking examples of the nontrivial behavior that strongly interacting electronic systems may display. ${ }^{3,4}$ Finite systems like quantum dots (QD's) provide simpler physical realizations of strongly interacting electronic systems where different models can be tested. When they are submitted to strong magnetic fields, the projection of the system to the lowest Landau level (LLL) becomes a good approximation which greatly simplifies theoretical studies in general and, in particular, makes exact diagonalization calculations feasible. Much work has been done on single QD's in the LLL regime yielding a reasonable understanding of the nature of their IS's. ${ }^{5,6}$ The search for IS's with well-defined properties which may produce a fractional QHE that is experimentally observable led to analyses of double-layered systems. ${ }^{7-12}$ Double quantum dots (DQD's) in a vertical configuration submitted to strong magnetic fields provide a finite system in which the existence of IS's is expected. However, the additional degree of freedom, together with the two new parameters-namely, the distance between the dots and the tunneling strength-may give rise to new phenomenology. For instance, Yang et al. ${ }^{12}$ suggest an experiment to test the quantum coherence of a special stable two-level system built in a DQD submitted to an adjustable interlayer bias voltage, which demonstrates suitable conditions for serving as quantum computing bits. Moreover, correlation effects can be experimentally detected in the far-infrared range (FIR) using uniform electric fields with nonvanishing component along the vertical direction as the generalized Kohn theorem, under such a condition, does not apply. ${ }^{13}$

This paper is organized as follows. In Sec. II we describe the model used in our calculation and analyze the Hamil- tonian of the system. In Sec. III, after identification of the incompressible states of interacting electrons, we begin with a review of the results previously obtained for single dots and show next our main results for double dots, which cover a wide range of input parameters. Finally, in Sec. IV we compare our findings with previous results in the literature and draw our conclusions.

\section{HAMILTONIAN}

We consider two identical two-dimensional quantum dots (in a vertical configuration) confined to the $X Y$ plane by equal parabolic potentials and submitted to a strong magnetic field directed along an arbitrary direction. The Hamiltonian of the system reads

$$
H=H_{0}+H_{t}+H_{e-e},
$$

where $H_{0}$ is the single-particle part which contains the kinetic contribution, the confining potential, and the Zeeman term. We adjust the input parameters in such a way that Landau level mixing is negligible. Then, in secondquantization formalism is given by

$$
H_{0}=\alpha M+\beta N-\Delta_{Z} S,
$$

where

$$
\begin{gathered}
\alpha=\frac{\hbar}{2}\left(\sqrt{\omega_{c}^{2}+4 \omega_{0}^{2}}-\omega_{c}\right), \\
\beta=\frac{\hbar}{2} \sqrt{\omega_{c}^{2}+4 \omega_{0}^{2}},
\end{gathered}
$$

and

$$
\Delta_{Z}=\mu_{B} g B,
$$

$\omega_{0}$ being the confining potential frequency, $\omega_{c}$ the cyclotron frequency given by $\omega_{c}=e B / m^{*} c$ ( $m^{*}$ is the effective elec- 
tron mass, $B$ the magnetic field, and $e$ and $c$ the electron charge and the speed of light in vacuum, respectively), $\mu_{B}$ $=e \hbar / 2 m c$ the Bohr magneton, and $g$ the Lande factor (we will consider $|g|=0.44$ whenever the Zeeman term is included). $M=\sum_{i=1}^{N} m_{i} a_{\sigma_{i}}^{\dagger} a_{\sigma_{i}}$ is the total angular momentum, and $N$ is the total number of electrons. $a_{\sigma_{i}}^{\dagger}$ creates a singleparticle state, and $\sigma_{i}$ refers to the three indexes that characterize the single-particle wave functions: angular momentum, spin, and isospin ( $s$ or $a$ associated with symmetric and antisymmetric combinations of wave functions concentrated in each dot: right and left). The tunneling term is given by

$$
H_{t}=-\frac{\Delta_{t}}{2} X
$$

where $\Delta_{t}$ is the energy gap between the symmetric and antisymmetric states in the noninteracting system and $X=N_{S}$ $-N_{A}$ is given by the balance between symmetric and antisymmetric states. Finally the two-body interaction part of the Hamiltonian is given by

$$
\begin{gathered}
H_{e-e}=\frac{1}{2} \sum_{i j k l} \sum_{\Lambda=1}^{3} V_{i j k l}^{(\Lambda)} a_{\sigma_{i}}^{\dagger} a_{\sigma_{j}}^{\dagger} \alpha_{\sigma_{l}} a_{\sigma_{k}}, \\
V_{i j k l}^{(\Lambda)}=\left\langle i j\left|V^{(\Lambda)}\right| k l\right\rangle,
\end{gathered}
$$

where the index $\Lambda$ is used to distinguish between the three different possibilities: (i) $V^{(1)}=0$ when only one change of a single-particle isospin takes place, (ii) $V^{(2)}=\frac{1}{2}\left(V_{r r}+V_{r l}\right)$ when both isospins remain unchanged, and (iii) $V^{(3)}$ $=\frac{1}{2}\left(V_{r r}-V_{r l}\right)$ when both isospins are changed. ${ }^{13} V_{r r}$ and $V_{r l}$ are the intradot and interdot Coulomb potentials, respectively, which are given by

$$
V_{r r}=\frac{e^{2}}{\epsilon r}
$$

and

$$
V_{r l}=\frac{e^{2}}{\epsilon\left(r^{2}+d^{2}\right)^{1 / 2}},
$$

$d$ being the distance between the dots along the $z$ direction, $\vec{r}$ a two-dimensional vector, and $\epsilon$ the dielectric constant of the host semiconductor. We have assumed Dirac $\delta$ distributions along the $z$ direction and have taken as a basis Slater determinants built up from Foch-Darwin single-particle wave functions projected on the LLL. ${ }^{5}$ The diagonalization can be performed in separated subspaces characterized by three well-defined quantum numbers: the total angular momentum $M$, the total spin $S$ along the direction of the field $\vec{B}$, and the parity $P$ related to the reflection symmetry with respect to the plane midway between the dots $\left[P\right.$ defined as $P=(-1)^{X / 2}$ for even $N$ and $P=(-1)^{(X+1) / 2}$ for odd $\left.N\right]$. We will define the set $(M, S, P)$ as a configuration.

The eigenstates within each configuration are determined by $H_{e-e}+H_{t}$ alone, and the role of the constant term given by $H_{0}$ is to shift the eigenenergies as a whole without changing their relative order.

\section{INCOMPRESSIBLE STATES IN THE LLL}

\section{A. Single QD}

Before studying the IS's in DQD's, we briefly review previous work on single QD's and its consequences. For a QD an IS with total energy $E$ and characterized by $(M, S)$ is identified as the one which has the following singular property $^{14}$ : the lowest excited state with quantum numbers $(M+1, S)$ has energy $E+\alpha$. That is to say, the energetically most favorable way to excite an IS increasing its total angular momentum by one unit is by moving the system as a whole-namely, by increasing by one unit the angular momentum of the center of mass (c.m.) only and leaving the internal structure unchanged. This characteristic was nicely recognized analyzing the Coulomb contribution to the total energy of a full polarized QD as a function of $M$. A periodical arrangement of plateaus (steplike structure) in the otherwise decreasing curve signaled the values of the magic angular momenta. ${ }^{14}$ Furthermore, the variation of the magnetic field (or the confining potential) did not drive the ground state (g.s.) through all neighboring values of $M$ but through the sequence of magic values only. ${ }^{15}$

This scenario corresponds to the regime characterized by a filling factor lower or equal to 1 , defined as ${ }^{14}$

$$
\nu=\frac{N(N-1)}{2 M},
$$

which involves the minimum possible value of the total angular momentum for a full polarized QD given by $M_{\min }$ $=N(N-1) / 2$ (the "compact state") and the angular momentum $M$ of the magic state. Some care must be taken for low values of $B$ for which the assumption of the LLL regime is not fulfilled. A suitable way to check this condition is by making sure that the energy of the highest single-particle occupied state is much smaller than $\omega_{+}=(\hbar / 2)\left(\sqrt{\omega_{c}^{2}+4 \omega_{0}^{2}}\right.$ $\left.+\omega_{c}\right)$, which is the energy gap between Landau levels for noninteracting electrons. The filling factor refers to the number of sublevels occupied within the LLL. There are two sublevels (spin up and down) in the case of a single QD and four (two for spin and two for isospin) in the case of a DQD. In general, for regimes in which several sublevels are occupied, the filling factor of a QD is not well defined. g.s.'s which are not related to IS's are also possible under such multiple-sublevel occupancy.

The sequence of magic filling factors depends on the number of electrons; for $N=3$ the values of $\nu$ are $\nu$ $=1, \frac{1}{2}, \frac{1}{3}, \frac{1}{4}, \ldots$ or for $N=4$ they are $\nu=1, \frac{3}{5}, \frac{3}{7}, \frac{1}{3}, \ldots$, in both cases related to the magic angular momentum given by

$$
M=\frac{1}{2} N(N-1)+j N,
$$

where $j$ is a positive integer number. It turns out that analysis of the Coulomb contribution to the total energy as a function of $M$ gives exhaustive and precise information about the magic values of the angular momentum and hence about the magic filling factors. The magic values of $M$ are the initial values of the plateaus. However, no information about the 


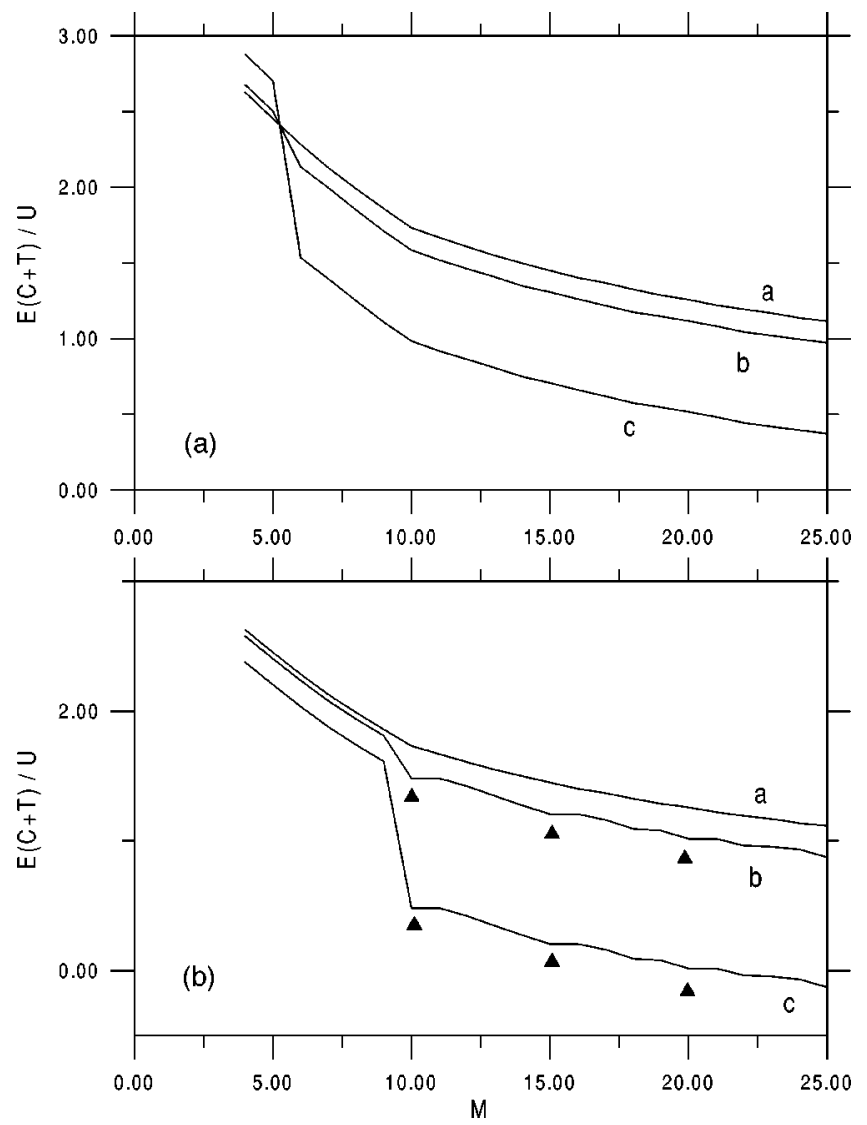

FIG. 1. (a) Coulomb plus tunneling contribution to the total energy as a function of $M$ for $N=5, S=N / 2$, and parity $P=1$, for several values of the tunneling gap: $\Delta_{t}=0$ (curve $a$ ), $\Delta_{t}$ $=2.2 \mathrm{meV}$ (curve $b$ ), and $\Delta_{t}=11 \mathrm{meV}$ (curve $c$ ). (b) The same as (a) for $P=-1$. The triangles point to the beginning of the plateaus. We have taken $B=5 \mathrm{~T}, d=20 \AA$, and $\hbar \omega_{0}=2.6 \mathrm{meV}$.

total spin of the IS's comes from the previous analysis. In the $N=3$ case, for a QD, the sequence of g.s.'s is always full polarized $\left(S_{z}=3 / 2\right)$ if the Zeeman term is included in the Hamiltonian (with $|g|=0.44$ ) or, in contrast, oscillations between $S_{z}=3 / 2$ and $S_{z}=1 / 2$ were obtained if no Zeeman term is included in the calculation. ${ }^{14,15}$ However, in the last case, the changes in spin and angular momentum do not appear simultaneously.

\section{B. DQD for $d \sim l_{B}$}

For a DQD we have a richer parameter space to be explored as, in addition to the parameters of a single QD, $\Delta_{t}$ and $d$ also enter the Hamiltonian, which open new possibilities for IS's to exist. We will focus on the phase diagram $\left(\Delta_{t} / d\right)$ for standard values of the remaining input parameters. Due to the fact that Coulomb interaction and changes in parity are coupled processes in a DQD, we define the "interaction" energy as the Coulomb plus the tunneling contribution $(C+T)$.

For $d \sim l_{B}$, where $l_{B}$ is the magnetic length given by $l_{B}$ $=\sqrt{\hbar / m^{*}\left(\omega_{c}^{2}+4 \omega_{0}^{2}\right)^{1 / 2}}$, the pure Coulomb contribution to the total energy ( $\alpha=\beta=\Delta_{t}=\Delta_{Z}=0$ ) as a function of $M$ is a decreasing function without plateaus as is shown in curves $a$

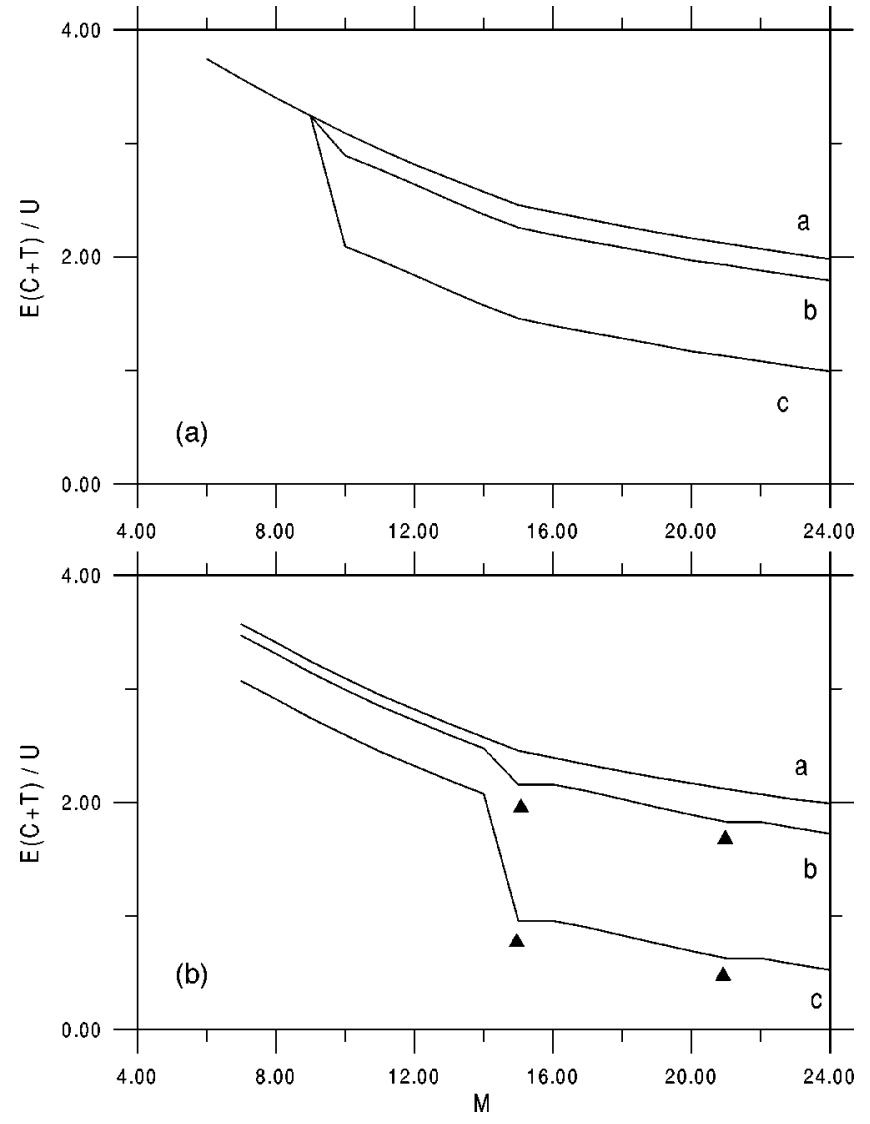

FIG. 2. The same as Fig. 1 for $N=6$.

in Figs. 1 and 2 for $N=5$ and $N=6$, respectively (energies are given in units of $u=e^{2} / \epsilon l_{B}$ ). Figures 1(a) and 2(a) correspond to parity $P=1$ and Figs. $1($ b) and $2($ b) to $P=-1$. All four cases refer to fully polarized systems $(S=5 / 2$ for $N=5$ and $S=3$ for $N=6$ ). For each value of $M$, the energy displayed is the lowest within the configuration $(M, S, P)$. The absence of plateaus can be understood as follows. Since $\Delta_{t}=0$, the number of electrons in each dot is a well-defined number. Hence, in order to increase the total angular momentum by one unit, the angular momentum of either dot must be increased by one unit, which unavoidably increases the typical distance from the electrons of one dot to the ones of the other dot and, therefore, decreases the interdot Coulomb energy.

According to Figs. 1 and 2 , it is necessary to include a sizable tunneling contribution in order to obtain a sequence of plateaus, which, furthermore, only occur for $P=-1$. Indeed, from a series of calculations for $N=5$ (not shown in Fig. 1), which correspond to a variation of $\Delta_{t}$ from 0 to 2 $\mathrm{meV}$ by small steps, we see a number of plateaus gradually appearing as $\Delta_{t}$ increases. We find that from $\Delta_{t}=2$ to 0.8 meV the sections from $M=10$ to $M=11$ and from $M=15$ to $M=16$ are exact plateaus. For $\Delta_{t}=0.4 \mathrm{meV}$ they are approximately flat and for $\Delta_{t}=0.2 \mathrm{meV}$ they disappear. However, in all cases the curves are abruptly decreasing before $M=10$ and between magic values. That is to say, we do not find any extra value of magic $M$ different from those given by Eq. (11). 


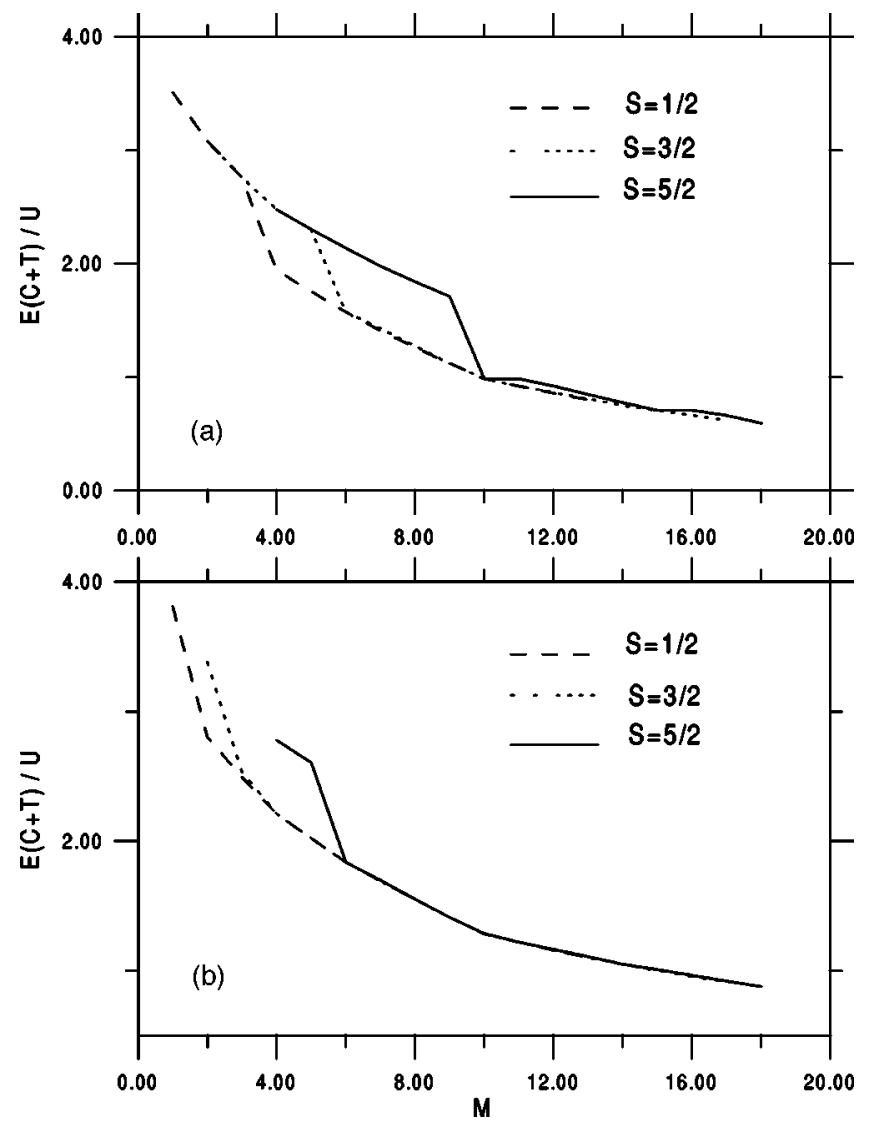

FIG. 3. (a) Coulomb plus tunneling contribution to the total energy as a function of $M$ for $N=5$ and parity $P=-1$ for all the possible values of the spin $S$. (b) The same as (a) for $P=1$. We have taken $B=5 \mathrm{~T}, d=20 \AA, \hbar \omega_{0}=2.6 \mathrm{meV}$, and $\Delta_{t}=2 \mathrm{meV}$.

In order to obtain information about the spin and parity of the IS's, we calculated the Coulomb plus tunneling contribution for all possible configurations. Figure 3 (for $N=5$ ) shows that the sequence of plateaus appear only when the system is fully polarized in spin and have parity $P=-1$ (similar results were obtained for $N=6$ ). Furthermore, although the parity $P=-1$ for $N=5$ can be obtained from different values of $X$-i.e., $X=N_{S}-N_{A}=5,1$, or -3 , the occupancy of the single-particle states for such incompressible g.s. turns out to be $X=5$ only; namely, the system is always fully spin and isospin polarized. This suggests that the g.s.'s that are IS's will not present variations in $S$ or $P$ as $B$ increases. This last suggestion was confirmed, for a Zeeman contribution different from zero, by an explicit calculation of the g.s. vs $B$, which turns out to be always fully spin and isospin polarized. Figure 4 displays the total energy of the g.s. as a function of magnetic field. The arrows point to the places where the angular momentum jumps from one magic value to the next one, leaving the spin and parity unchanged. In the inset we show $E_{\text {g.s. }}-\beta N$ in order to compare with other publications which omit the $N$-dependent term. The nearly monotonous function of $B$ is due to the fact that, in the absence of spin or isospin transitions, the interaction energy has a negligible influence in the plot and hence the evolution of the system is driven by the monotonous increasing term $\beta N$ which is much more important than the

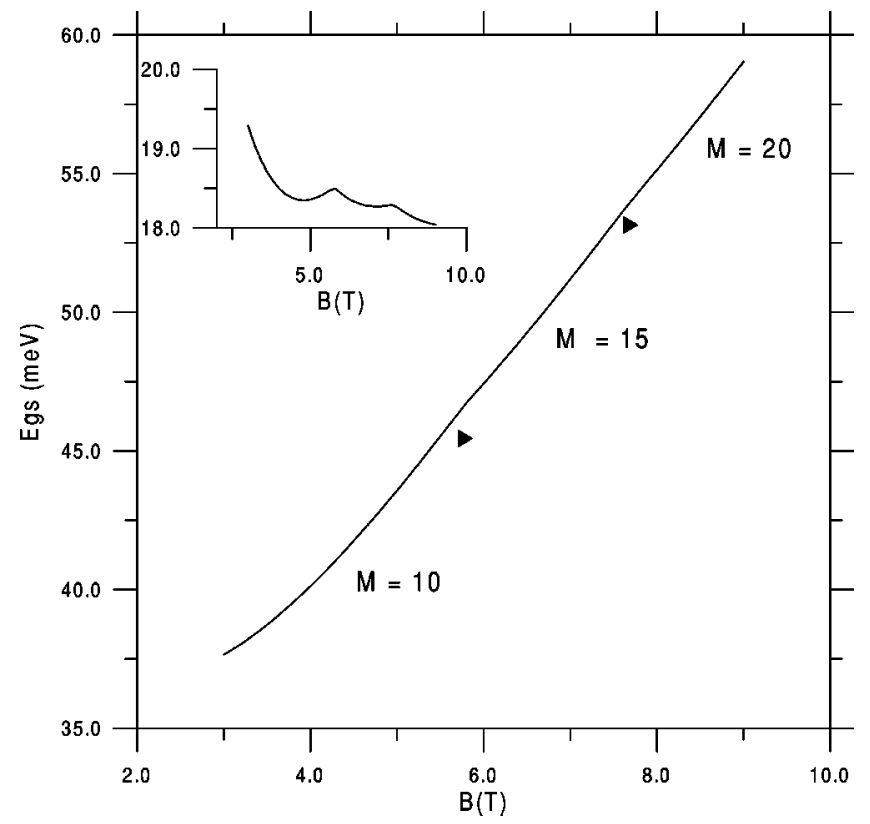

FIG. 4. Ground-state energy vs $B$ for $N=5$. The triangles point to the transitions between magic angular momenta. We use the same values for $d, \omega_{0}$, and $\Delta_{t}$ as in Fig. 3. Inset: $E_{g . s .}-\beta N$ as a function of $B$.

decreasing term $\alpha M$, which would produce kinks at the transition points, as is shown in the inset $\left(E_{\text {g.s. }}-\beta N\right.$ vs $\left.B\right)$. In brief, the full spin and isospin polarization appears to be a well-defined attribute of these IS's, which result from manybody effects.

As shown in Fig. 3(a) the interaction energy appears to be degenerated at the magic values with respect to the three possible spin polarizations. Since the curves that belong to $S=\frac{3}{2}$ and $\frac{1}{2}$ have lower energy at the end of the plateaus, the final balance of energy depends critically on the relation between this difference of interaction energy, the kinetic, and the Zeeman terms. That is to say, an IS that is the g.s. for a given value of $B$ and $\omega_{0}$ will remain as g.s. as $B$ increases or $\omega_{0}$ decreases only if

$$
E_{C+T}\left(M, \frac{3}{2}, 1\right)-E_{C+T}\left(M+1, \frac{3}{2}, 1\right)<\alpha+\Delta_{Z}
$$

or otherwise the new g.s. will be a compressible and not fully spin polarized state at $M+1$. Hence, as $B$ or $\omega_{0}$ changes, the g.s. can be driven into compressible zones in contrast with the results obtained for single QD's.

The single-particle occupancies of the $m$ values for the first three IS's for $N=5$, calculated at $B=4,7$, and $9 \mathrm{~T}$, respectively, are shown in Fig. 5. The first g.s. for $M=10$ $(\nu=1)$ is the compact fully polarized state which belongs to a one-dimensional subspace and, as a consequence, no correlation is involved as one Slater determinant produces the exact solution. Moreover, the density is a "dome"-shaped circular symmetric distribution without any structure. As $B$ grows, the angular momentum changes from $M$ to $M+N$; all the electrons jump together moving away from the origin, forming a ring. The dimension of the g.s. subspace increases 


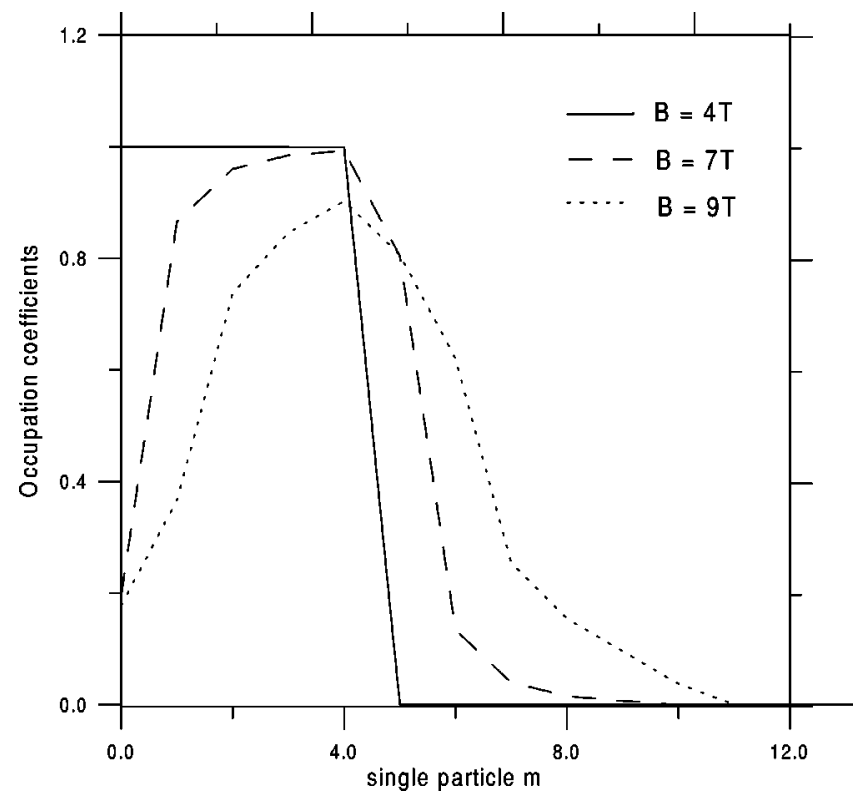

FIG. 5. Total occupancy of the single-particle angular momentum states $m$ for $N=5$. The values of $B$ considered correspond to the magic values $M=10,15$, and 20 , respectively. We have taken $d=20 \AA, \hbar \omega_{0}=2.6 \mathrm{meV}$, and $\Delta_{t}=6 \mathrm{meV}$.

and as the relative weights of the different Slater determinants within the expansion of the g.s. become significant for different elements of the bases; namely, the correlation becomes important.

\section{DQD: Phase diagram $\Delta_{t} / d$}

So far we have explored the situation $d \sim l_{B}$. In order to get the complete scenario of IS's in a DQD, we have also investigated in detail the remaining regions of the phase diagram $\left(\Delta_{t} / d\right)$.

In Figs. 6-9 we follow, for $N=3$, the variation of the interaction energy vs $M[E(C+T) / M]$ as $d$ and $\Delta_{t}$ change.

From $A$ to $B$ (Fig. 6), as was just discussed for the case $d \sim l_{B}$, the plateaus emerge as $\Delta_{t}$ grows from zero until they are well defined at $\Delta_{t}=0.11 u$; that is to say, for values of $M$ given by Eq. (11) the system evolves from compressible to incompressible states. For $d$ close to zero (at the left of point $B)$ and $\Delta_{t}$ large, all the electrons are in the symmetric state. As a consequence, the interaction energy of a single QD can be reproduced with high accuracy by the addition of the constant contribution $\Delta_{t} X / 2$ to the energy of the DQD in this region.

From $A$ to $D$ (Fig. 7), tunneling between the two dots is not allowed. Starting from a curve without plateaus for small distances $(d \sim 10 \AA$ ), we move across the transition regime with a gradual formation of new plateaus at $M=M_{R}+M_{L}$ where $M_{R}$ and $M_{L}$ are the magic numbers of single QD's. We come close to point $D$ at $d=1000 \AA$ which shows the features of two decoupled dots with $N=1$ (with no contribution to the Coulomb term) and $N=2$ (with magic numbers $M=1,3,5,7, \ldots$ ), respectively. This regime in which tunneling is forbidden has been previously studied for double layers, and special attention has been devoted to the $\nu=1$

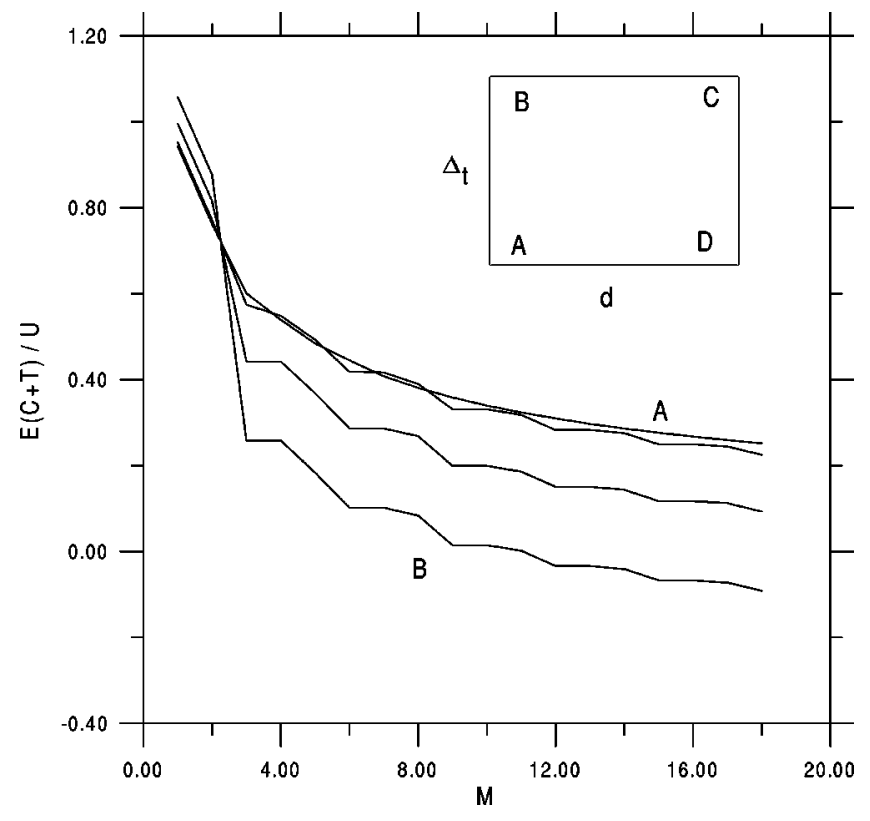

FIG. 6. Coulomb plus tunneling contribution vs $M$ for $N=3$. From $A$ to $B$ the tunneling gap is $0.0,0.018 u, 0.106 u$, and $0.229 u$, respectively. Inset: phase diagram used. We have taken $\hbar \omega_{0}$ $=2.6 \mathrm{meV}, B=15 \mathrm{~T}, d=10 \AA, S=3 / 2$, and $P=1\left(l_{B}=65 \AA\right)$.

case. ${ }^{7,8,12}$ For a double layer the incompressible state $\nu=1$ is observed for values of $d$ about the magnetic length. ${ }^{8}$ Furthermore, as $d$ increases, the state exhibits a phase transition to a compressible one. The difference between the two cases comes from the fact that, as was previously discussed, when $\Delta_{t} \sim 0 M_{R}$ and $M_{L}$ are well-defined quantum numbers, the increase by one unit of $M$ means the increase of $M_{R}$ or $M_{L}$ (but not both), changing, in a DQD, the relative position of

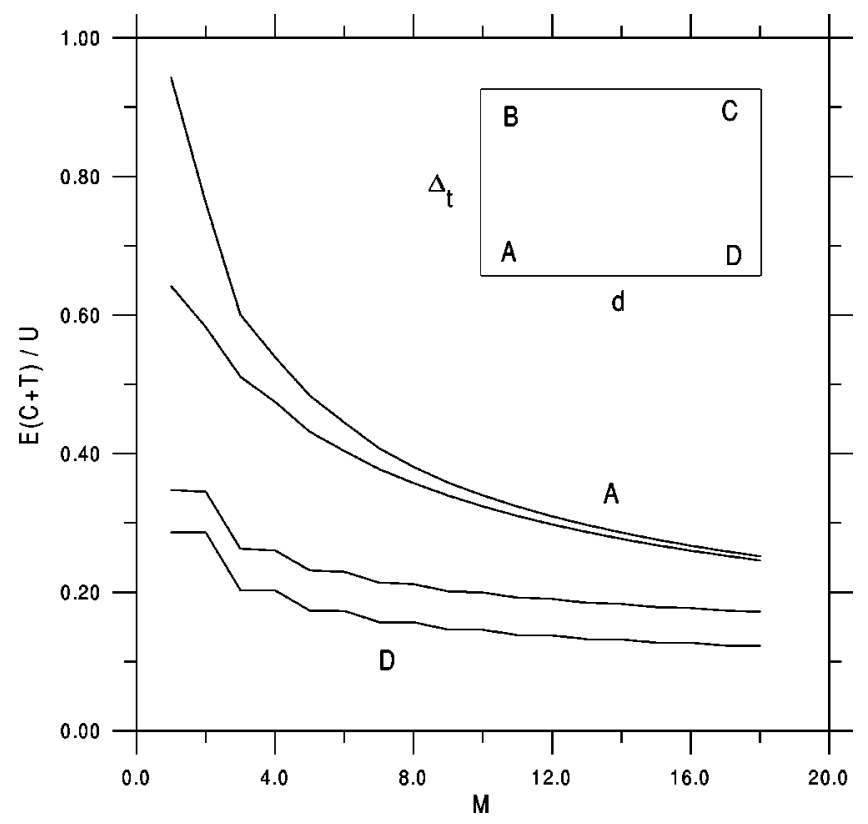

FIG. 7. The same as Fig. 6 for $\Delta_{t}=0$. From $A$ to $D$ the interdot distance is: $10,50,500$, and $1000 \AA$, respectively. Inset: phase diagram used. 


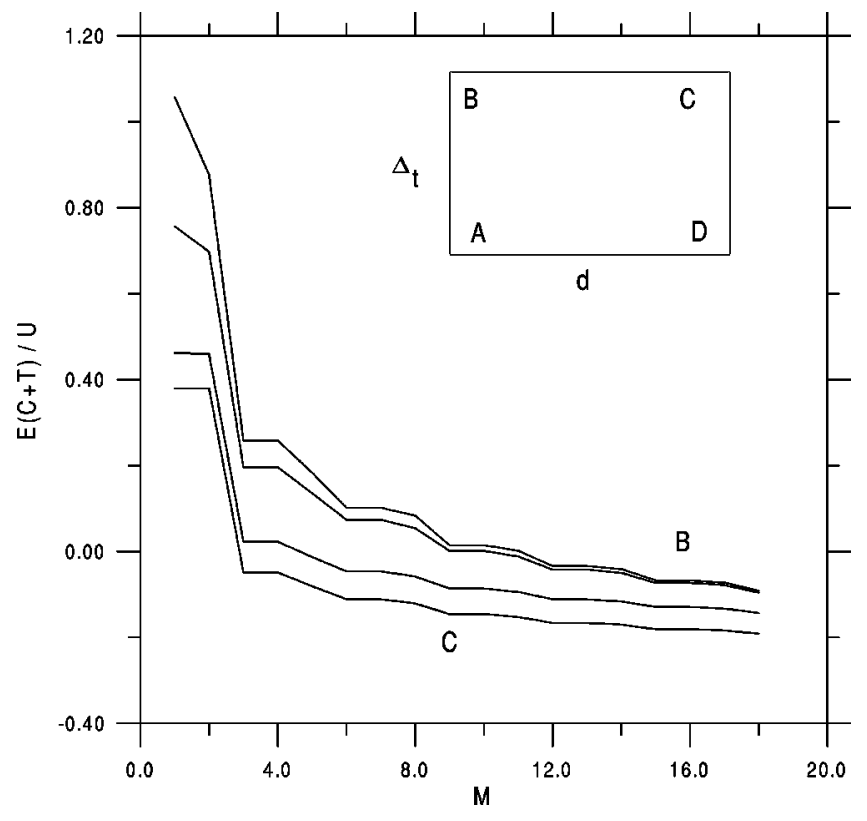

FIG. 8. The same as Fig. 6 for $\Delta_{t}=0.229 u$. From $B$ to $C$ the interdot distance is 50, 100, 500, and $1000 \AA$, respectively. Inset: phase diagram used.

the electrons in each dot and so decreasing the Coulomb interaction which prevents the formation of a plateau. However, this is not the case for a double layer in which the shift of charge due to the change of angular momentum does not change the relative interlayer distribution of charge, allowing for the appearance of plateaus. For large $d$ the two layers decouple and hence one would expect (for total $\nu=1$ ) two $\nu=1 / 2$ IS's. However, since a fractional QHE of $\nu=1 / 2$ is not observable for a single layer, these states were not identified in Ref. 8.

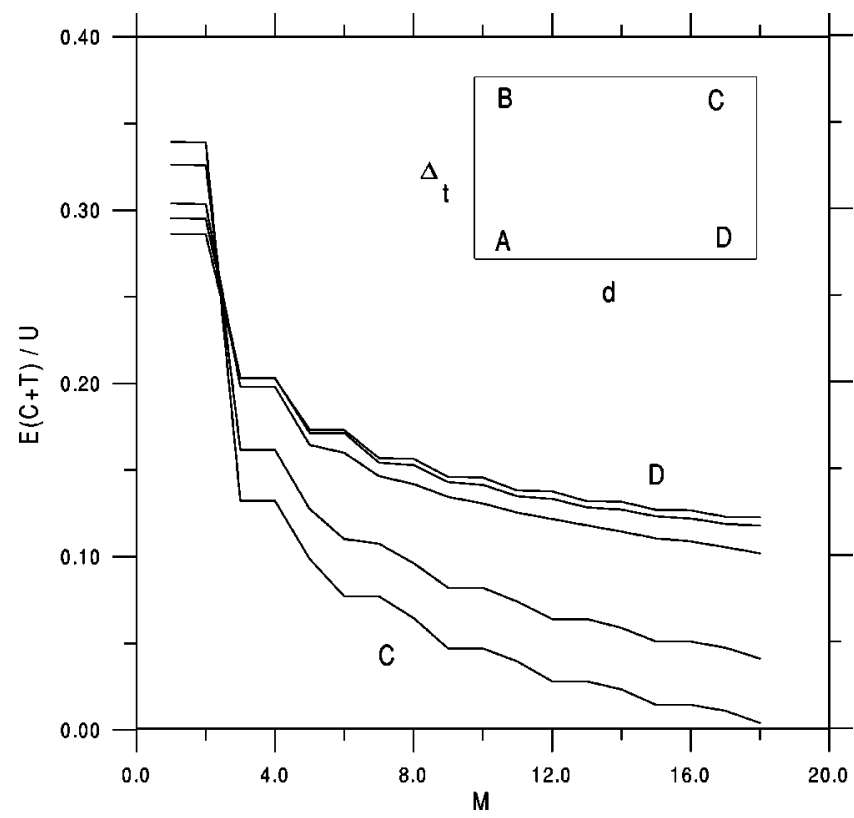

FIG. 9. The same as Fig. 6 for $d=1000 \AA$. From $D$ to $C$ the tunneling gap is $0.0,0.018 u, 0.035 u, 0.080 u$, and $0.106 u$, respectively. Inset: phase diagram used.
From $B$ to $C$ (Fig. 8), although the distance $d$ grows, the sequence of magic numbers typical of a DQD does not disappear due to the relative large value of $\Delta_{t}\left(\Delta_{t}=0.229 u\right)$. An exceptional case appears for $d>500 \AA$ at $M=1$ (for $N$ $=5$ the analog exceptional case appears at $M=4$ ). It turns out to be the only IS within the LLL regime which does not fulfill the general rule of being fully spin and isospin polarized. The subspace associated with the appropriate configuration [i.e., $(M=1, S=3 / 2, P=1)$ ] is one dimensional and the only Slater determinant in the bases has one electron in the symmetric state and two electrons in the antisymmetric state (or $X=-1$ ). It is the only IS with no single-dot analog. Our interpretation of the fact that the $M=1$ magic value appears only for relatively large distances is as follows: for large tunneling and small $d$, the system is closer to a single dot with $N=3$ than to a DQD of the same number of electrons (as discussed before). Thus $M=1$ can only appear when the Coulomb interdot interaction weakens related to $\Delta_{t}$ and DQD properties different from those of a single QD may arise. Notice, however, that for the $M=1$ state to be a g.s. such a low magnetic field (or large confining potential) is required that the LLL regime assumption would not apply anymore. Finally, even for values of $d$ as large as $1500 \AA$ (being $t_{B}=65 \AA$ ), we did not find the transition from DQD to two decoupled single dots.

From $D$ to $C$ (Fig. 9), the tunneling increases and the system of two decoupled QD's with a period of two typical of the $N=2$ single dot evolves into a DQD, reproducing the period of 3 typical of an $N=3$ DQD. During the transition, there is a narrow interval of values of $\Delta_{t}$ for which the $E(C+T)$ vs $M$ curve has no plateaus (except for the $M=1$ case). That is to say, an initially incompressible g.s. would evolve into a compressible state and again into a IS as $\Delta_{t}$ increases. This evolution takes place as the system changes from two decoupled single dots to a DQD.

Compressible regions have been obtained before by Rontani et al. ${ }^{16}$ for DQD with finite width. They consider the evolution of the g.s. of the system of $N=6$ as $d$ increases for $\Delta_{t}$ exponentially decreasing with $d$, which is equivalent to the evolution along a trajectory from $B$ to $D$ in our phase diagram. They obtain a small zone of compressibility in the middle, related to the transition from a regime where the system behaves as a unique coherent system to a regime of well-separated QD's. We observe the same qualitative behavior along the $B-C$ trajectory (which is different to theirs), although we do not obtain the same magic values.

The transition from DQD to two decoupled single QD's as $\Delta_{t}$ decreases has been observed before by Partoens and Peeters ${ }^{10}$ by means of a current spin density functional calculation.

We have also studied the $D$ to $C$ evolution for $N=5$ which shows the same qualitative behavior. As $D$ is approached the structure of plateaus can be understood in terms of the IS of two decoupled $N=2$ and $N=3$ single QD's, although the analysis is much more intricate than for the $N$ $=3$ case.

\section{DISCUSSION}

We have investigated in detail the existence of IS's that result from the Coulomb many-body effects in a DQD for the entire phase diagram $\left(\Delta_{t} / d\right)$. 
An important point in our analysis is the criterium used to identify IS's. First we want to emphasize that in contrast to the case of a single layer for which the integer QHE is associated with gaps of single-particle origin and the fractional QHE is associated with gaps involving many-body effects, for double-layered systems (and thus in accordance for DQD), single-particle as well as many-body regimes can be related to the QHE at the same filling factor by the tuning of appropriate sample parameters. ${ }^{8}$ The IS's we are interested in are those associated with $e-e$ interactions (coupled with tunneling) and thus signaled somehow in the variation of the interaction energy with $M$. As discussed in the previous section, we define the interaction energy as the Coulomb plus tunneling contributions and require IS's to preserve the interaction energy when the angular momentum $M$ is increased by one unit. We want to stress that this is not equivalent to identifying magic $M$ from the kinks of the lowest energies of each configuration as a function of $M$ or from the kinks of the variation of the absolute g.s. energy as a function of $B$, as has been used in the literature ${ }^{17,18}$ to identify correlated IS's.

Our criterium is equivalent to the one used by Laughlin in Ref. 1 for the single layer, as we discuss below. The Hamiltonian is separable into the c.m. and the relative coordinates and, as a consequence, the total angular momentum can be analyzed as $M=M_{c . m .}+M_{r e l}$ and the energy as $E_{t o t}=E_{c . m}$. $+E_{\text {internal }}$. For three two-dimensional electrons, Laughlin obtains that the internal energy as a function of the relative angular momentum has downward cusps at special (magic) values $(M=3,6,9,12, \ldots)$. These magic values appear to be related to incompressibility: the area of the system defined as the area of the triangle determined by the correlated positions of the electrons within these states changes discontinuously as pressure is applied. At the downward cusps, $E_{\text {internal }}\left(M_{\text {rel }}\right)<E_{\text {internal }}\left(M_{\text {rel }}+1\right)$ for the lowest-energy states of each configuration. They are the only states for which the increase of $M_{r e l}$ by one unit requires a positive amount of internal energy. In order to show that our criterium $^{14}$ is equivalent to Laughlin's, ${ }^{1}$ notice first that $E_{C+T}(M)$ only depends on $M_{r e l}$ and $M_{r e l} \leqslant M$. Since $E_{C+T}(M)$ is defined as the minimal energy among those of the states with total angular momentum $M$, it implies that it is the minimal energy among all states with relative angular momentum $M_{r e l} \leqslant M$. Hence, given $M$ and $E_{C+T}(M-1)$, $E_{C+T}(M-1) \neq E_{C+T}(M) \quad$ implies $E_{C+T}(M-1)$ $>E_{C+T}(M)$ and furthermore $M=M_{\text {rel }}$. Since $E_{C+T}$ reduces to $E_{\text {internal }}$ for a single layer, if $E_{C+T}(M-1)>E_{C+T}(M)$, then $E_{\text {internal }}\left(M_{\text {rel }}-1\right)>E_{\text {internal }}\left(M_{\text {rel }}\right)$ for $M_{\text {rel }}=M$. Namely, negative slopes in our plots imply negative slopes in Laughlin's. If, on the contrary, $E_{C+T}(M-1)=E_{C+T}(M)$, then $M_{r e l}$ has not changed and $M$ contains at least one unit of c.m. angular momentum. $E_{C+T}(M)$ being the minimal energy with total angular momentum $M$, it implies that any state with $M_{r e l}=M$ has larger energy than $E_{C+T}(M-1)$. If, in addition, $E_{C+T}(M-2)>E_{C+T}(M-1)$, as is always the case in our plots, then $M-1$ contains only relative angular momentum, as shown above. Then, for the single layer, $E_{C+T}(M-1)=E_{C+T}(M) \quad$ implies $\quad E_{\text {internal }}\left(M_{\text {rel }}-1\right)$ $<E_{\text {internal }}\left(M_{\text {rel }}\right)$ for $M_{\text {rel }}=M$. Namely plateaus in our plots imply positive slopes in Laughlin's, which concludes our

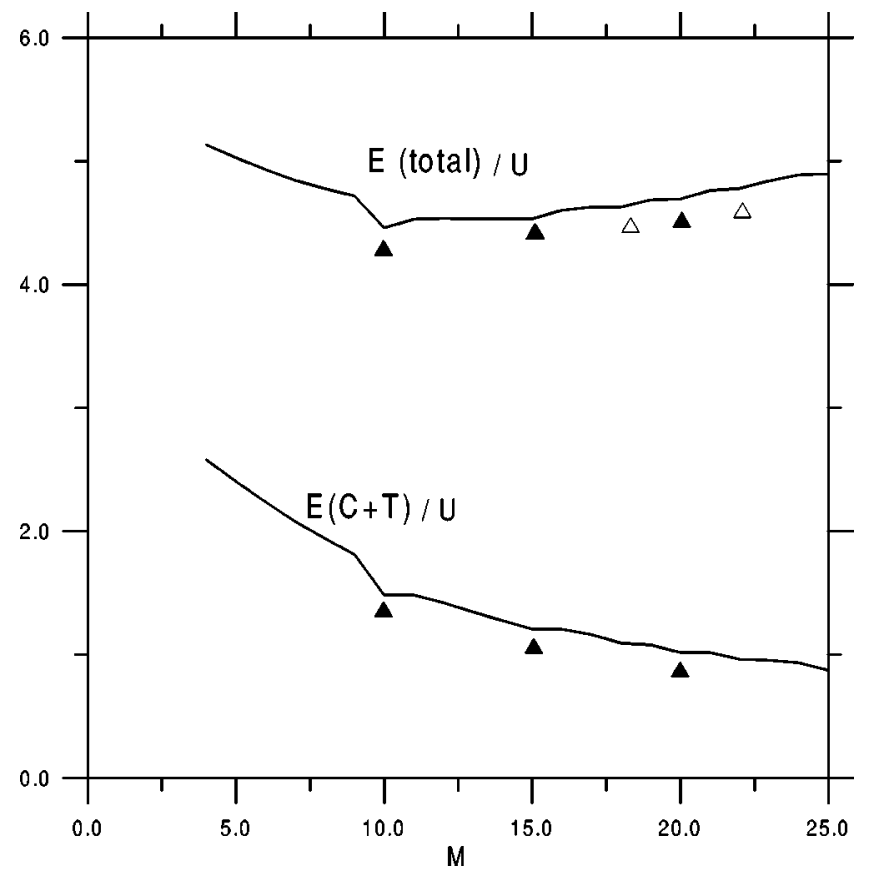

FIG. 10. Coulomb plus tunneling contribution (lower curve) and total energy (upper curve) as a function of $M$ for $N=5$. The solid triangles point to the actual magic values $M$ whereas the open triangles point to cusps which could be mistaken by them. The values of $B, \omega_{0}, d$, and $\Delta_{t}$ are the same as in Fig. 3 .

proof. On the other hand, there is no guarantee that downward cusps in the curve $E_{t o t}$ vs $M$ are related to plateaus in the $E_{C+T}$ vs $M$ curve. It is enough to have $\alpha>E_{C+T}(M)$ $-E_{C+T}(M+1)$ at a nonmagic $M$ to obtain there a downward peak in $E_{\text {tot }}$ vs $M$ which is not related to a plateau in $E_{C+T}$ vs $M$.

For $d \sim l_{B}$ the fact that the magic $M$ follow Eq. (11) is in conflict with the claim made in Refs. 17 and 18 that extra magic values for $M$ (depending on the value on the tunneling strength) exist in this regime. The authors of Refs. 17 and 18 identified IS's with downward cusps of the total energy as a function of $M$-i.e., the interaction energy (our curve) with the addition of the single-particle contribution. In Fig. 10 we show the two possibilities for $N=5$. It is clear from the upper curve that some downward cusps, which would be identified as IS's by the criterium of Refs. 17 and 18, do not actually correspond to IS's in our criterium.

In order to make sure that the discrepancies with Refs. 17 and 18 are only due to the different criteria to identify IS's, we have reproduced their results (see Fig. 11). To be more precise, we performed the calculation for $N=3$ and the same input parameters as those used in Ref. $18(N=3, B=15 \mathrm{~T}$, $d=200 \AA, \hbar \omega_{0}=3 \mathrm{meV}$, and $\Delta_{t}=0.2 \mathrm{meV}$ ). In Fig. 11(a) the interaction energy contribution versus $M$ is shown. Due to the low value of the tunneling contribution, the plateaus that will appear, for larger values of $\Delta_{t}$, at $M=3,6$, and 9 are still not visible and the only ones that already appear are $M=12$ and 15 . If the kinetic contribution is added, it comes out that the g.s. is at $M=5$ [see Fig. 11(b)] at the lowest 


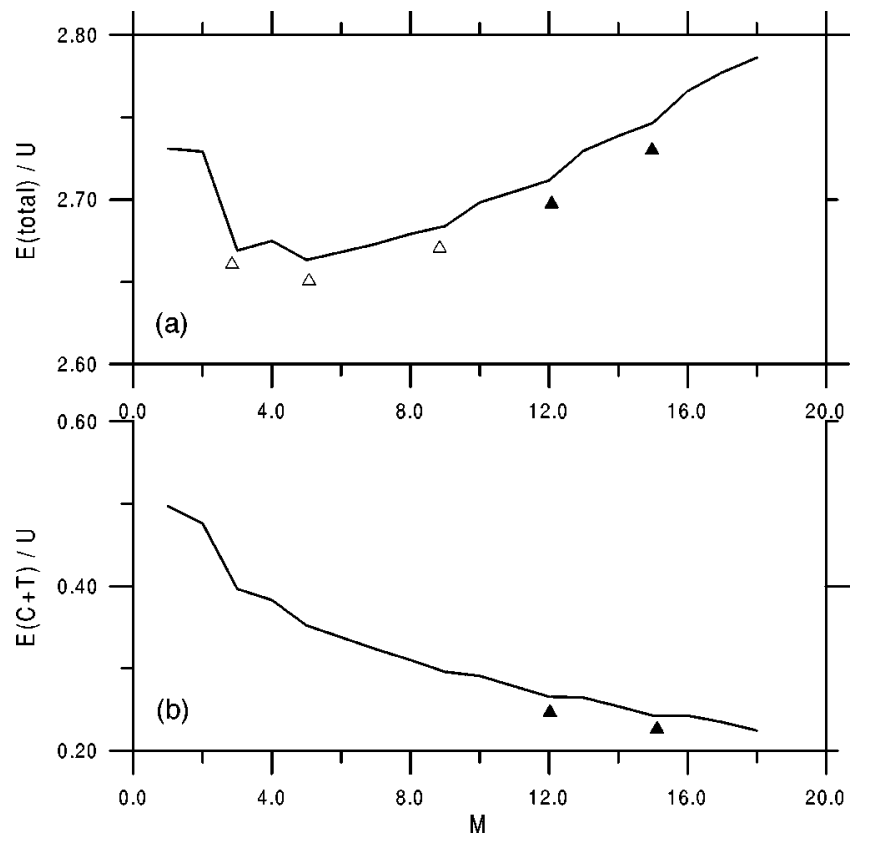

FIG. 11. (a) Coulomb plus tunneling contribution vs $M$ for $N$ $=3$. (b) The same as in (a) for the total energy. The input parameters are given in the text. The solid triangles point to the actual magic values $M$ whereas the open triangles point to cusps which could be mistaken by them.

downward cusp of the total energy in accordance with Ref. 18.

A word of caution should be given here as a number of relevant papers exist in the literature ${ }^{18-21}$ which use the term magic angular momentum to denote the angular momentum $M$ which displays downward cusps in the curve $E_{\text {tot }}(M)$. The corresponding states enjoy enhance stability and have been the subject of intensive studies. In particular Refs. 19 and 20 provide characterizations of these states ranging from small values of $M$, where the fractional quantum Hall regime is sometimes identified, to large values of $M$, where striplike structures and Wigner molecules seem to appear (see Ref. 21 for a review and Ref. 22 for related work on layers). However, only a subset of these states fulfills our criterium of incompressibility and the magic $M$ displayed in Eq. (11) corresponds to this subset only.

Our conclusions can be summarized as follows.

(i) The downward cusps obtained by Laughlin in Ref. 1 turn out to be equivalent to the plateaux of the curve $E_{C+T}$ vs $M$.

(ii) All the incompressible states are fully spin and isospin polarized (except the $M=1$ case for $N=3$ ). Since a single QD fully spin polarized and a DQD fully spin and isospin polarized are systems with no extra degrees of freedom aside from angular momentum, we expect a similar behavior for the electronic distance quantization as that obtained in Ref. 1.

(iii) An exceptional incompressible state was found for $d / l_{B} \geqslant 8$ at $M=1$ and $X=-1$ for $N=3$ and $M=4$ and $X$ $=1$ for $N=5$. This is the only one that is not fully isospin polarized and does not have a single QD analog. However, for it to be a g.s. values of the input parameters that do not fulfill the assumption of the LLL regime are required.

(iv) For $d \sim l_{B}$, it is not possible to obtain IS's if the tunneling is small.

(v) For small $\Delta_{t}$, as $d$ grows, the DQD evolves into two decoupled single QD's. New magic values appear which correspond to the addition of magic numbers of two decoupled QD's.

(vi) An IS at $M$ will remain as the g.s. under changes of $B$ and/or $\omega_{0}$ if the condition

$$
E_{C+T}(M)-E_{C+T}(M+1)<\alpha+\Delta_{Z}
$$

is fulfilled. In general, however, the variation of $B, \omega_{0}$, or $d$ can drive the g.s. from incompressible into compressible zones of the phase diagram. This behavior differs from the well-known properties of a single QD for which the variation of the g.s. as $B$ or $\omega_{0}$ changes is driven through incompressible states only, skipping all nonmagic values of $M$.

(vii) Whenever the g.s. is fully spin and isospin polarized, it is an IS. In other words, the IS's are the only possible g.s. for $\nu$ lower than 1. However, for $\nu>1$ other g.s.'s are possible.

Before closing, let us briefly elaborate on the last point. Notice that the following situations are also possible: (a) g.s.'s with $M$ that fulfills Eq. (11) and are not IS's due to the fact that from $M$ to $M+1$ there is no plateau. This condition can be obtained for very low values of $\Delta_{t}$, for example, for $N=5, M=10, B=5 \mathrm{~T}, \hbar \omega_{0}=2.6 \mathrm{meV}, \Delta_{t}=0.2 \mathrm{meV}$, and $d=20 \AA$. (b) Ground states with $M$ not given by Eq. (11) for which the system is not fully spin or isospin polarized (or $\nu>1)$ and $E(C+T)$ has not a constant evolution from $M$ to $M+1$. This is the case for example for $N=5, M=13$ $\left(B=6 \mathrm{~T}, \quad \hbar \omega_{0}=2.6 \mathrm{meV}, \quad d=20 \AA, \quad\right.$ and $\quad \Delta_{t}=5.86$ $\left.\times 10^{-3} \mathrm{meV}\right)$. In the last case, $S=N / 2$ and $P=-1$; however, $X \neq N$.

Notice also that the previously discussed states are not the only possible g.s.'s within the LLL regime. For instance, if the confining potential is strong enough, other types of g.s.'s are possible like the ferromagnetic, canted, and symmetric states (all of them with $\nu=2$ ) first studied in double layers ${ }^{23}$ and latter recognized in DQD. ${ }^{24}$

Finally, let us note that the correlation plays an increasingly important role as the magnetic field grows up. The interaction energy and correlation effects can be experimentally tested by uniform electric fields with nonvanishing component along the $z$ direction due to the fact that under this condition, the Kohn theorem does not apply ${ }^{13}$ and the FIR spectroscopy becomes sensitive to the internal structure.

\section{ACKNOWLEDGMENT}

We gratefully acknowledge C. Tejedor and L. MartínMoreno for the code used for the Hamiltonian diagonalization. This work has been performed under Grants No. BFM2002-01868 from DGESIC (Spain), No. FPA2001-3598 from MCyT and Feder (Spain), and No. 2001GR-0064 and No. 2001SGR-00065 from Generalitat de Catalunya. 
${ }^{1}$ R.B. Laughlin, Phys. Rev. B 27, 3383 (1983).

${ }^{2}$ R.B. Laughlin, Phys. Rev. Lett. 50, 1395 (1983).

${ }^{3}$ The Quantum Hall Effect edited by R.E. Prange and S.M. Girvin (Springer-Verlag, New York, 1990).

${ }^{4}$ D. Yoshioka, The Quantum Hall Effect (Springer-Verlag, Berlin, 2002).

${ }^{5}$ L. Jacak, P. Hawrylak, and A. Wjs, Quantum Dots (SpringerVerlag, Berlin, 1998).

${ }^{6}$ T. Chakraborty, Quantum Dots (North-Holland, Amsterdam, 1999).

${ }^{7}$ T. Chakraborty and P. Pietiläinen, Phys. Rev. Lett. 59, 2784 (1987).

${ }^{8}$ S.Q. Murphy, J.P. Eisenstein, G.S. Boebinger, L.N. Pfeiffer, and K.W. West, Phys. Rev. Lett. 72, 728 (1994).

${ }^{9}$ B. Partoens and F.M. Peeters, Phys. Rev. Lett. 84, 4433 (2000).

${ }^{10}$ B. Partoens and F.M. Peeters, Europhys. Lett. 56, 86 (2001).

${ }^{11}$ E. Anisimovas and F.M. Peeters, Phys. Rev. B 65, 233302 (2002).

${ }^{12}$ S.-R. Eric Yang, J. Schliemann, and A.H. Mac Donald, Phys. Rev. B 66, 153302 (2002).

${ }^{13}$ N. Barberán and J. Soto, Phys. Rev. B 66, 205325 (2002).
${ }^{14}$ P.A. Maksym and T. Chakraborty, Phys. Rev. Lett. 65, 108 (1990).

${ }^{15}$ P. Hawrylak and D. Pfannkuche, Phys. Rev. Lett. 70, 485 (1993).

${ }^{16}$ M. Rontani, G. Goldoni, F. Manghi, and E. Molinari, Europhys. Lett. 58, 555 (2002).

${ }^{17}$ H. Imamura, P.A. Maksym, and H. Aoki, Phys. Rev. B 53, 12613 (1996).

${ }^{18}$ H. Imamura, P.A. Maksym, and H. Aoki, Phys. Rev. B 59, 5817 (1999).

${ }^{19}$ T. Seki, Y. Kuramoto, and T. Nishino, J. Phys. Soc. Jpn. 65, 3945 (1996).

${ }^{20}$ C. Yannouleas and U. Landman, Phys. Rev. B 66, 115315 (2002).

${ }^{21}$ P.A. Maksym, H. Imamura, G.P. Mallon, and H. Aoki, J. Phys.: Condens. Matter 14, R299 (2000).

${ }^{22}$ N. Shibata and D. Yoshioka, J. Phys. Soc. Jpn. 72, 664 (2003).

${ }^{23}$ S. Das Sarma, S. Sachdev, and L. Zheng, Phys. Rev. B 58, 4672 (1998).

${ }^{24}$ L. Martin-Moreno, L. Brey, and C. Tejedor, Phys. Rev. B 62, R10 633 (2000). 